\title{
Study on Magnetic Properties of Electric Magnetic Materials Under External Temperature
}

\author{
Jiang Wenyu ${ }^{1}$, Li Weiye ${ }^{2}$, Lou Jianyong ${ }^{1}{ }^{*}$, Wu Shidong ${ }^{3}$ \\ ${ }^{1}$ State Key Laboratory of Electrical Insulation and Power Equipment, Xi'an Jiaotong University, Xi'an, China \\ ${ }^{2}$ CRRC Zhuzhou Locomotive Co., Ltd., Zhuzhou, China \\ ${ }^{3}$ Wuxi Putian Core Co., LTD, Wuxi, China
}

Email address:

jylou@mail.xjtu.edu.cn (Lou Jianyong), jiangwenyu@stu.xjtu.edu.cn (Jiang Wenyu)

${ }^{*}$ Corresponding author

\section{To cite this article:}

Jiang Wenyu, Li Weiye, Lou Jianyong, Wu Shidong. Study on Magnetic Properties of Electric Magnetic Materials Under External Temperature. Science Discovery. Vol. 6, No. 3, 2018, pp. 205-211. doi: 10.11648/j.sd.20180603.21

Received: April 11, 2018; Accepted: May 17, 2018; Published: June 26, 2018

\begin{abstract}
For electrical manufacturers, with the development of the power industry, the demand for special electrical equipment, such as large capacity transformers and high temperature transformers, is becoming more and more. The magnetic properties of silicon lamination at different temperatures have a very important reference value for designers to design products that meet the relevant parameters. Firstly, this paper selects various brands of electrical silicon lamination, designs and makes different test samples. Secondly, this paper adopts the ring sample method of one dimension magnetic property measurement, and makes the measurement of the magnetic property parameters under different temperature conditions, obtains the related test data and the change of the magnetic characteristic parameters of test samples and uses data processing method to sort out the test data. Finally, this paper analyzes the reasons for the data. The results show that magnetic properties have certain regularity with the change of temperature, and the measurement method in this paper can serve for practical engineering.
\end{abstract}

Keywords: Silicon Lamination, Different Temperatures, Magnetic Properties Measurement

\section{外加温度影响下电工磁性材料磁特性研究}

\author{
姜文宇 ${ }^{1}$, 李伟业 ${ }^{2}$, 娄建勇 ${ }^{1 *}$, 吴世东 ${ }^{3}$ \\ ${ }^{1}$ 电力设备电气绝缘国家重点实验室, 西安交通大学, 西安, 中国 \\ ${ }^{2}$ 中车株洲电力机车有限公司, 株洲, 中国 \\ ${ }^{3}$ 无锡普天铁心股份有限公司, 无锡, 中国
}

\section{邮箱}

jylou@mail.xjtu. edu. cn(娄建勇), jiangwenyu@stu.xjtu. edu. cn(姜文宇)

\begin{abstract}
摘要: 对于电器生产厂家来说, 随着电力行业的发展, 大容量变压器、耐高温变压器等特殊电气设备的需求也越来越 大，硅钢片在不同温度下的磁特性参数对设计者们设计出满足相关指标参数的产品具有非常重要的参考价值。本文首 先选取了多种牌号的电工硅钢片, 设计制作了不同的测试样品, 同时采用了一维磁特性测量中的环样测试法, 对试样 做了在不同温度条件下磁特性参数测量, 获取了相关测试数据, 得到了测试样品磁特性参数的变化情况, 利用数据拟 合的处理方法, 对测试数据进行整理, 最后对数据出现的原因进行了分析。结果表明: 磁特性随温度变化具有一定规 律性，本文的测量方法能够为实际工程服务。
\end{abstract}


关键词: 电工硅钢, 不同温度, 磁特性测量

\section{1. 引言}

随着电能的开发和利用, 世界范围内电力生产事业正 处于黄金发展时期, 特高压、大容量等多种电磁设备的制 造也迎来了发展的良机, 如何能精确设计和制造这些电磁 设备, 需要设计者了解电磁设备中电磁材料在实际工况下 准确的磁性能参数, 所以, 对与电力生产事业发展戚戚相 关的磁性材料进行实际工况下的磁性能参数的准确测量 具有非常重要的意义 [1-2]。

近几十年以来, 磁性材料的磁特性测量方面的工作在 各国学者做了大量的研究工作之后取得了一定的成果。关 于二维磁特性测量方面, 日本学者在多年前对于硅钢片二 维磁特性测量的研究就已经起步, 但是, 硅钢片的二维磁 特性在材料特性模拟过程中, 出现了许多问题, 尤其是磁 场强度轨道不对称, 这是现在磁特性测量的一个难点问题 [3]。中国沈阳工业大学于 2009 年组织召开了有关二维磁 特性测量方面的国际会议, 也取得了一些进展, 但就目前 而言, 在二维磁特性测量方面还没有能完成精确测量的仪 器, 也没有相应的检测标准 [4]。三维磁特性测量时实际 问题相当复杂, 目前有澳大利亚悉尼理工大学的朱建国在 这方面进行了初步的研究, 并制作了一个三维的测量模型, 但磁场均匀度的测量效果并不理想 [5-6]。研究者们对这 一问题目前处于探索阶段, 对其相应的应用于实际工程的 研究理论也停留在实验室层面。相对于一维磁性材料, 磁 性材料的磁特性测试技术相对完善, 由于一维磁特性测试 方法中的爱泼斯坦方圈法和环样测试法具有测量重复性 好、准确度高、测量速度快等优点 [7-8], 到目前为止, 它仍作为一种最为成熟的磁测试技术被广大生产商和用 户所采用 $[9-10]$ 。

由于研究方法的复杂性, 有关磁性材料测量方面仍有 很大发展空间。本文将以多种电工硅钢片材料为实验样品, 对试样进行不同温度条件下的磁特性参数测量, 并对数据 进行整理分析, 总结磁性材料磁特性参数变化规律。

\section{2. 磁特性测量方法}

电机、变压器、各种电气设备的铁芯, 都需要大量的 硅钢材料作为其主磁路, 而硅钢的磁性能优劣关乎到整个 磁性材料制作的电气设备的各项性能参数。目前对电工硅 钢片磁特性测量方法主要还是采用一维磁特性测试: 包括 爱泼斯坦方圈法和环样测试法, 在这里, 本文采用环样测 试法研究研究硅钢片处于不同温度下的磁特性的情况 [11]。

\section{1. 环样测试法}

环样测试法是一种一维磁性材料测试法, 它具有重复 性好、精度高等优点, 是一种比较常用的测试方法。环样 测试法的基本结构图如下图1所示。

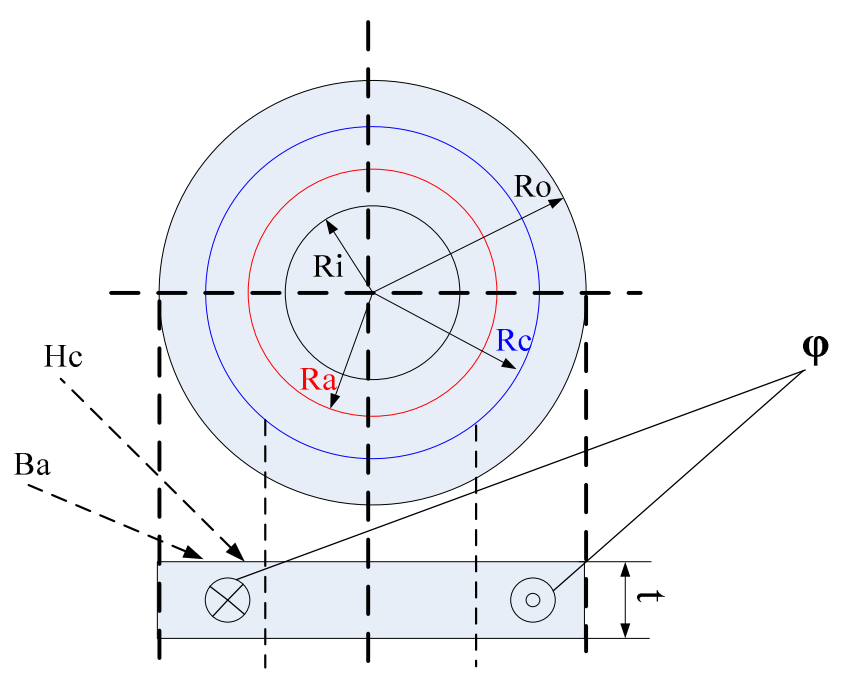

图1 环样测试结构图。

其测量原理如下:

设平均磁场强度为 $H_{a}$, 平均磁感应强度为 $B_{a}$, 通过 环样横截面的总磁通记为 $\Phi$ 。

式 (1) 为计算磁感应强度的公式:

$$
B_{a}=\frac{\Phi}{\left(r_{o}-r_{i}\right) d}
$$

其中, 环样的内、外半径分别记作 $r_{i}$ 和 $r_{o}$, 环样的厚 度记为 $d$ 。

平均磁通密度与平均磁场强度之间的关系如下:

$$
H_{a}=f\left(B_{a}\right)
$$

磁场强度的测量方法类似爱泼斯坦方圈法, 都是利用 采用电阻法得到电流, 然后通过相应的计算公式得到磁场 强度的大小, 如式 (3) 所示:

$$
H_{a}=N I / 2 \pi r_{a}
$$

式中:

$N$ 一励磁线圈的匝数;

$I$ 一流过励磁线圈的电流;

$r_{a}$ 一平均磁路长度对应的半径。

\section{2. 数据处理方法}

本文的数据处理方法, 是在利用环样测试法测得数据 的基础上, 通过寻找发现和整理数据, 对实验数据用数学 公式进行拟合, 发现磁特性参数的规律, 从而计算出试验 测量中未测量的点或者能够预测实验仪器量程之外的部 分 [12]。由此可以看出, 如何能够获取近似测量的数据的 
拟合多项式尤其重要。通过以下实验数据近似的方法来求 得数学公式。

实验过程中得到的实验数据, 可以将其看成是平面曲 线上的一组点, 但要描述此曲线的函数通常情况下都非常 复杂, 所以, 一般情况下, 采用数据近似的方法, 即寻找 一个比较准确且易于计算的近似函数来表示平面曲线上 那一组点, 使得寻找的函数具有与数据反映的规律具有相 似性, 这样利用该函数就可以反应磁性材料的相关特性。

假设给定平面上 $m+1$ 个数据点 $\left\{\left(x_{i}, y_{i}\right)\right\}(i=0,1,2, \ldots, m)$ 和一个函数 $P(x), P(x)$ 是一组 基 函 数 $g_{k}(x)(k=0,1,2, \ldots, n)$ 的函数, 使按某种标准, $P(x)$ 与数 据点接近。

$$
P(x)=k_{0} g_{0}(x)+k_{1} g_{1}(x)+\cdots+k_{n} g_{n}(x)
$$

以 $p_{i}=P\left(x_{i}\right)(i=0,1,2, \ldots, m)$ 记函数 $P(x)$ 在 $x_{i}$ 处的函 数值, 可以用式 (5) 、式 (6)、式 (7) 来度量接近的 程度。

$$
\begin{gathered}
E_{\infty}=\max _{i}\left|p_{i}-y_{i}\right| \\
E_{2}=\sqrt{\sum_{i=0}^{m}\left(p_{i}-y_{i}\right)^{2}} \\
E_{1}=\sum_{i=0}^{m}\left|p_{i}-y_{i}\right|
\end{gathered}
$$

若函数 $P(x)$ 按照近似标准式 (5)、式 (6) 和式 (7) 都能达到值为零, 即:

$$
P\left(x_{i}\right)=p_{i}=y_{i}, \quad i=0,1,2, \ldots, m
$$

满足式 $(8)$ 条件的函数 $P(x)$ 称为插值数据点 $\left\{\left(x_{i}, y_{i}\right)\right\}$ 的插值函数。本文对测试数据进行多项式拟合, 得到函数 是 $n$ 次多项式:

$$
P(x)=\sum_{j=0}^{n} k_{j} x^{j}
$$

插值条件式为:

$$
k_{0} x_{0}+k_{1} x_{i}+k_{2} x_{i}^{2} \cdots+k_{n} x_{i}^{n}=y_{i}
$$

式 (10) 是一个 $\mathrm{n}+1$ 个未知数 $k_{0}, k_{1}, k_{2} \ldots, k_{n}, m+1$ 个方 程的线性方程组, 其矩阵公式为:

$$
\left[\begin{array}{ccccc}
1 & x_{0} & x_{0}^{2} & \cdots & x_{0}^{n} \\
1 & x_{1} & x_{1}^{2} & \cdots & x_{1}^{n} \\
\vdots & \vdots & \vdots & & \vdots \\
1 & x_{n} & x_{n}^{2} & \cdots & x_{n}^{n}
\end{array}\right]\left[\begin{array}{l}
k_{0} \\
k_{1} \\
\vdots \\
k_{n}
\end{array}\right]=\left[\begin{array}{l}
y_{0} \\
y_{1} \\
\vdots \\
y_{n}
\end{array}\right]
$$

这样, 现在的问题就归结为如何求解现行方程组 (11)。 当 $m>n$ 是, 方程组 (11) 可能会无解。当 $m\langle n$ 时, 方程组 (11) 可能有多个解。因此, 在此讨论 $m=n$ 的情况。

$$
V=\left[\begin{array}{ccccc}
1 & x_{0} & x_{0}^{2} & \cdots & x_{0}^{n} \\
1 & x_{1} & x_{1}^{2} & \cdots & x_{1}^{n} \\
\vdots & \vdots & \vdots & & \vdots \\
1 & x_{n} & x_{n}^{2} & \cdots & x_{n}^{n}
\end{array}\right]
$$

该矩阵的行列式为:

$$
\operatorname{det}(V)=\prod_{p=1}^{n}\left[\prod_{q=0}^{p-1}\left(x_{p}-x_{q}\right)\right]
$$

只要 $x_{p} \neq x_{q}(p \neq q), \operatorname{det}(V) \neq 0$, 方程组（11）有唯 一解。从而由式 (9) 唯一确定一个 $n$ 次多项式 $P(x)$, 满 足插值条件式。

\section{3. 温度影响下硅钢片的磁特性测试}

随着磁性材料的不断发展, 电力行业对特种变压器、 大容量耐高温变压器等变压器设备的需求也越来越大, 硅 钢片在不同温度下的磁特性参数的好坏直接关系到设计 的产品是否符合相关指标参数 [13], 因此, 温度影响下的 硅钢片磁特性测试具有非常重要的参考价值, 本文进行了 硅钢片不同温度下的磁性能参数的测量和分析工作。本文 首先进行硅钢片在工频不同温度条件下的磁特性参数测 量, 然后对测量数据进行处理和分析。

\section{1. 实验样品与实验仪器}

TD8100磁性材料测试系统; 温度控制箱; (如图2所示)

选取牌号为30AQ130和23JGH的硅钢片环样测试品; 航空高温导线, 线径为 $0.75 \mathrm{~mm}$;

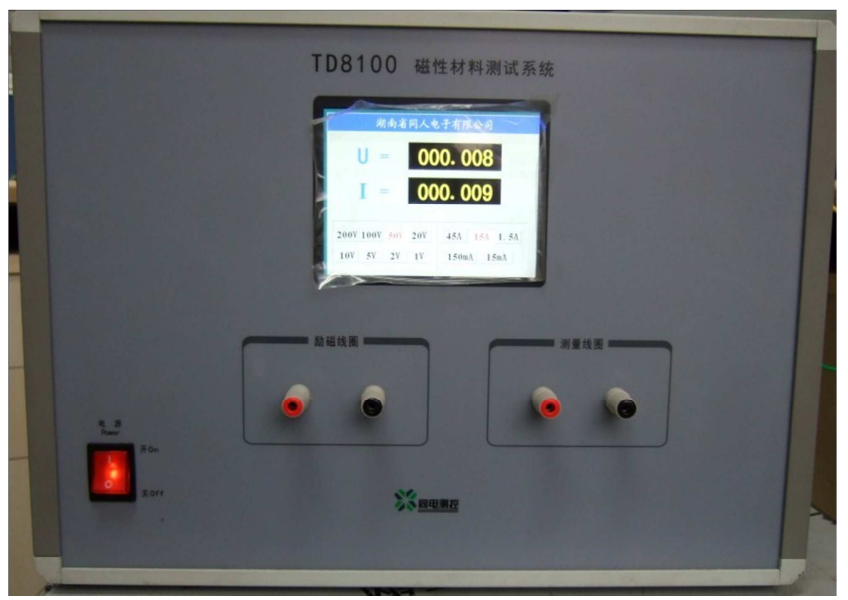

TD8100磁性材料测试系统 


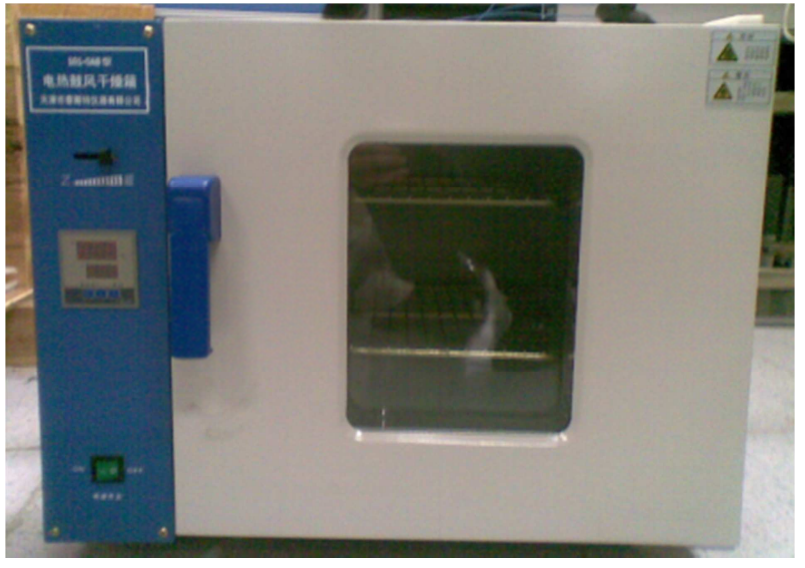

温度控制箱

图2 实验仪器。

\section{2. 硅钢片磁特性在不同温度下参数测量实验}

打开箱门, 将牌号为30AQ130和23JGH的实验样品放入 温度控制箱内, 关好箱门, 按照实验仪器操作规程设置好 实验参数, 调整实验温度, 将温度设置到预定测试值, 待 温度控制箱提示箱内温度达到设定值之后, 就可以开始实 验测试。在TD8100型硅钢片测量系统的测量主界面上用鼠 标点击 “一般测试” 或 “曲线测试”，系统将自动对先前 设置好的测试点开始测试。
本文温度影响下硅钢片磁特性测试参数的测试实验, 分别测试了样品在 $30^{\circ} \mathrm{C} 、 40^{\circ} \mathrm{C} 、 50^{\circ} \mathrm{C}, 60^{\circ} \mathrm{C} 、 70^{\circ} \mathrm{C}, 80^{\circ} \mathrm{C} 、$ $90^{\circ} \mathrm{C}, 100^{\circ} \mathrm{C} 、 110^{\circ} \mathrm{C}, 120^{\circ} \mathrm{C} 、 130^{\circ} \mathrm{C}, 140^{\circ} \mathrm{C} 、 150^{\circ} \mathrm{C}$, $160^{\circ} \mathrm{C}, 170^{\circ} \mathrm{C}$ 下: 损耗测试点 $0.9 \mathrm{~T} 、 1.0 \mathrm{~T} 、 1.1 \mathrm{~T} 、 1.2 \mathrm{~T}$ 、 $1.3 \mathrm{~T} 、 1.4 \mathrm{~T} 、 1.5 \mathrm{~T} 、 1.6 \mathrm{~T} 、 1.7 \mathrm{~T}$ 和 $1.8 \mathrm{~T}$ 。

\section{3. 实验数据后处理}

\subsection{1. 损耗P}

实际工况中, 电工磁性材料在电磁设备中应用时, 所 处的工作环境很多时候不是在标准工况中, 例如电工材料 由于比总损耗的存在, 会发热使得仪器设备的温度随之升 高, 温度升高导致工作环境变化, 所以, 拥有这些电磁材 料在不同温度下的比总损耗测试值, 对于生产厂家来说, 具有重要意义。

本文正是考虑到实际情况, 外界温度的改变导致影响 到制作设备的磁性材料的磁性能参数的变化, 通过模拟温 度变化, 测试样品的比总损耗, 经过实验得到了测试点在 $0.9 \mathrm{~T}-1.8 \mathrm{~T}$ 情况下, 实验温度从 $30^{\circ} \mathrm{C}$ 以 $10^{\circ} \mathrm{C}$ 递增到 $170^{\circ} \mathrm{C}$, 测试多组数值, 最终, 得到了相关的实际比总损耗汇总数 据。选取牌号为 $30 \mathrm{AQ} 130$ 的硅钢片数据。

1）不同B值

为了更直观的看出比总损耗随不同温度及测试点的 变化情况, 对数据进行图形处理。可以得到不同测试点下 比总损耗随温度变化的关系曲线如图3所示:

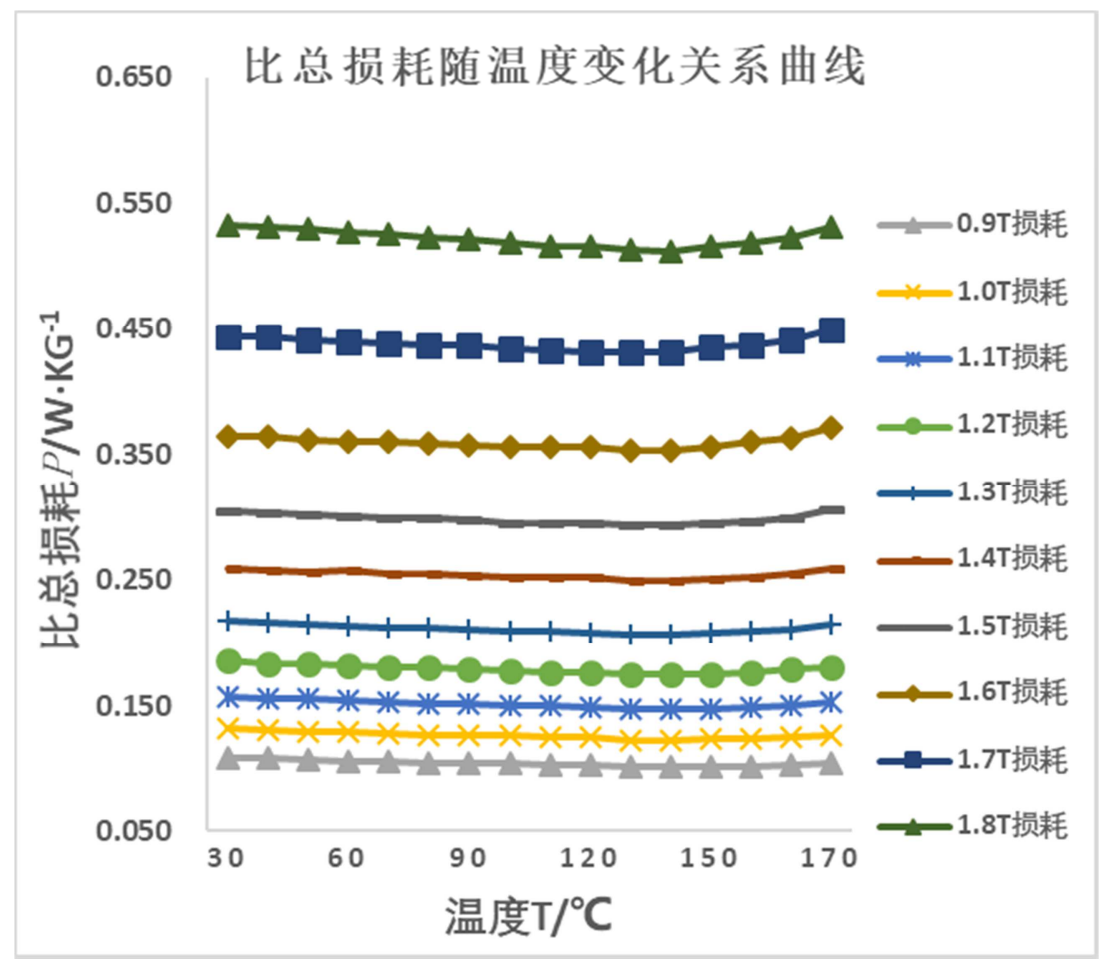

图3 比总损耗变化关系曲线。

当温度为 $30^{\circ} \mathrm{C}-130^{\circ} \mathrm{C}$ 时, 随着温度的增加, 其比总损 耗有减少的趋势; 这一现象产生的原因, 当温度达到这个 范围内, 样品的磁化强度随着温度的升高急速降低, 磁性 材料自身磁化强度减弱甚至为零, 会导致磁滞比总损耗减 少, 使得比总损耗值变小;
当温度为 $130^{\circ} \mathrm{C}-170^{\circ} \mathrm{C}$ 时, 其比总损耗相比原来略微 开始回升, 这种现象的可能原因是测试样品的胶水在 $130^{\circ} \mathrm{C}$ 以上时开始融化, 导致测试样品间的衔接发生了变 化, 从而影响了比总损耗的变化。 
从测试点方面考虑, 同一温度下, 对应的测试点的比 总损耗随着B值的增加也会随着增加, 而且这种趋势也跟 着变大。

\section{2) 不同温度}

分析比总损耗在不同温度的下的大小, 可绘出不同温 度的比总损耗曲线如图4所示, 为了方便总结规律, 故选 取了 $30^{\circ} \mathrm{C} 、 130^{\circ} \mathrm{C}$ 和 $170^{\circ} \mathrm{C}$ 下三个损耗曲线如下图5所示:

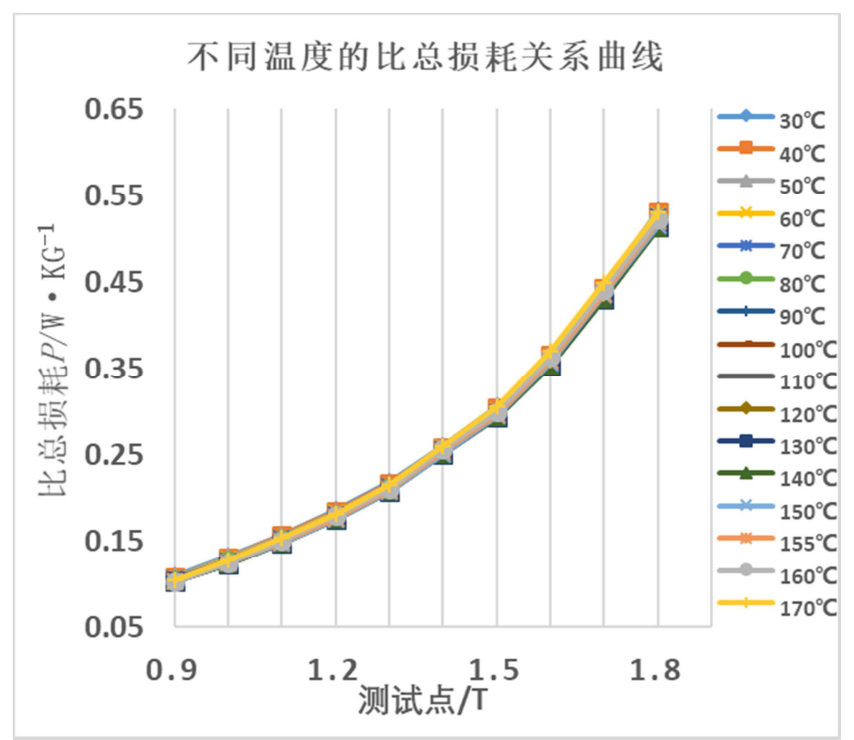

图4 不同温度下的比总损耗曲线。

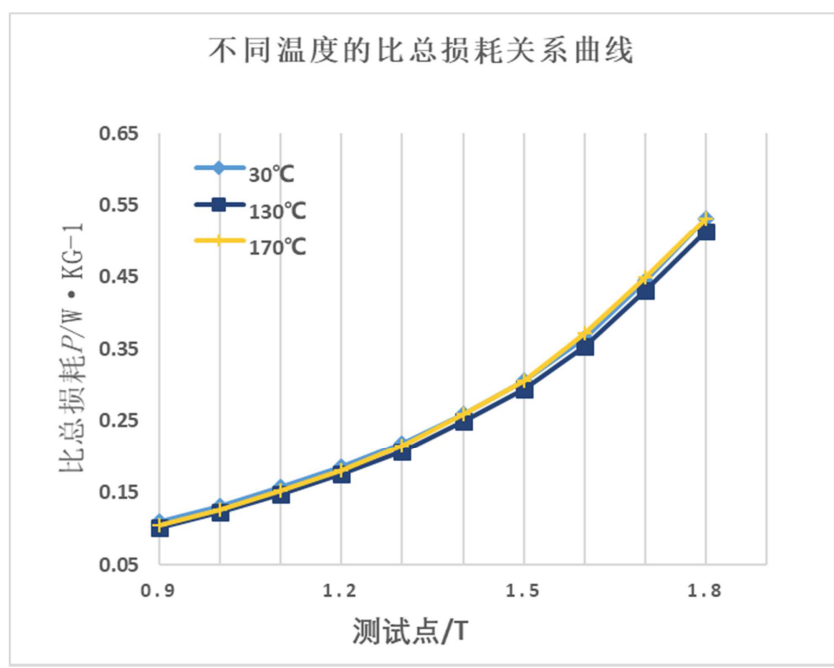

图5 $30^{\circ} \mathrm{C} 、 130^{\circ} \mathrm{C}$ 和 $170^{\circ} \mathrm{C}$ 下的比总损耗曲线。

由上图可以看出, $30^{\circ} \mathrm{C} 、 130^{\circ} \mathrm{C}$ 和 $170^{\circ} \mathrm{C}$ 的比总损耗曲 线变化幅度是基本一致, 且可以看出 $30^{\circ} \mathrm{C}$ 下的比总损耗大 于 $130^{\circ} \mathrm{C}$ 时的比总损耗, $130^{\circ} \mathrm{C}$ 下的比总损耗小于 $170^{\circ} \mathrm{C}$ 时 的比总损耗, 这就意味着, 随着温度的升高, 损耗是先降 低后升高的。由计算方法中多项式拟合相关知识, 可以得 到 $30^{\circ} \mathrm{C}$ 和 $170^{\circ} \mathrm{C}$ 的拟合曲线及拟合多项式。

根据多项式拟合方法可以求出上图中 $30^{\circ} \mathrm{C}$ 损耗 曲线的拟合多项式如式（14）所示:

$$
\begin{gathered}
y=0.2612 x^{3}-0.6624 x^{2}+0.7703 x-0.2377 \\
R^{2}=0.9997
\end{gathered}
$$

同理, 可以通过同样的拟合方法求得 $170^{\circ} \mathrm{C}$ 时损耗曲 线对应的拟合多项式，根据拟合多项式可以得到相应的拟 合曲线, 对应的四次拟合多项式如式 (15) 所示:

$$
\begin{gathered}
y=0.2738 x^{3}-0.7084 x^{2}+0.8164 x-0.258 \\
R^{2}=0.9999
\end{gathered}
$$

同理, 可以求出 $30^{\circ} \mathrm{C}^{\sim} 170^{\circ} \mathrm{C}$ 内其他温度下的比总损耗 的拟合多项式从而画出拟合曲线，根据实际需求，可以根 据拟合的多项式和曲线进行比总损耗参数的估读, 为实际 工程应用服务。

\subsection{2. 磁导率u}

通过对 30AQ130的测试样品进行实验测试, 得出磁导 率关于温度的函数曲线。

1) 不同B值

为了方便分析, 将磁导率的与温度的测试数据, 绘成 曲线如下图6所示:

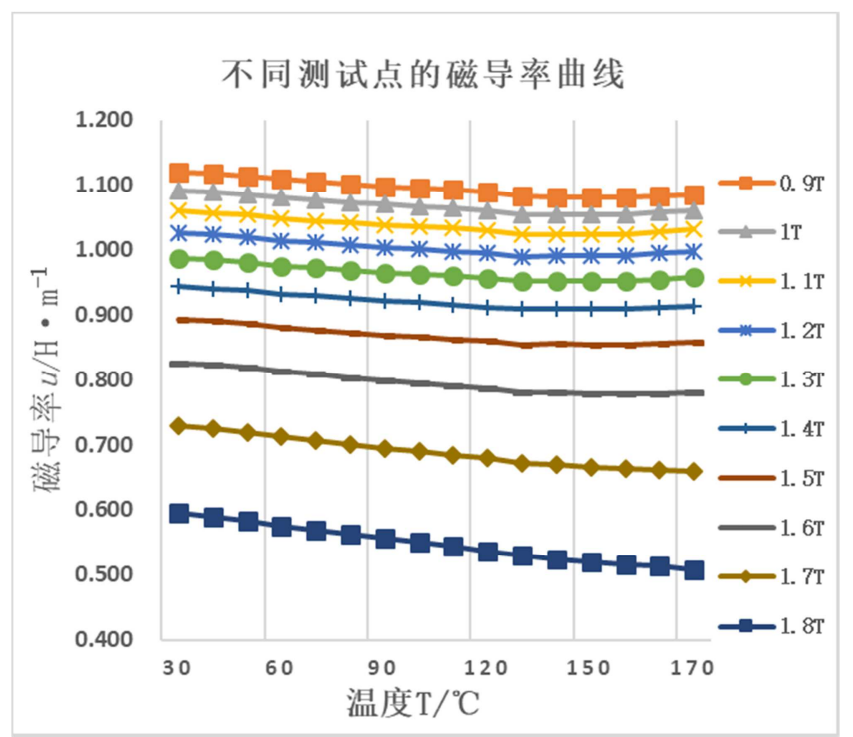

图6 不同测试点的磁导率曲线。

由上图可得, 测试样品的磁导率随着测试点 $\mathrm{B}$ 值的增 大而减小, 从1.3T左右开始, 这种减小的趋势更加明显。

对于同一个测试点来说, 曲线也并不是单调的, 可以 看出, 磁导率随着温度的升高具有先下降的趋势, 在 $150^{\circ} \mathrm{C}$ 左右曲线开始略微回升。为了进一步观察测试样品磁导率 随温度变化的情况, 做了如下曲线, 以便进一步分析。

2) 不同温度

根据实验所得数据, 可以得出不同温度的磁导率曲线, 方便于分析，如图7所示: 


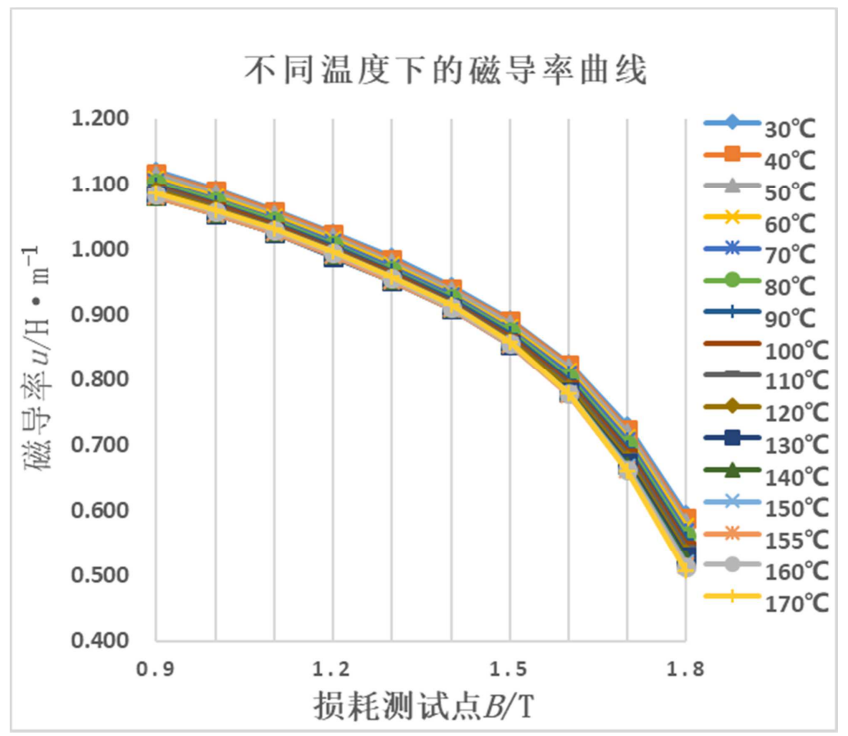

图7 不同温度的磁导率变化曲线。

从图可以得到, 磁导率总体上看随着温度的增加具有 下降的趋势。为了直观的进行比较, 现将三个边界曲线 $30^{\circ} \mathrm{C}, 150^{\circ} \mathrm{C}$ 和 $170^{\circ} \mathrm{C}$ 下的磁导率绘图为图8所示:

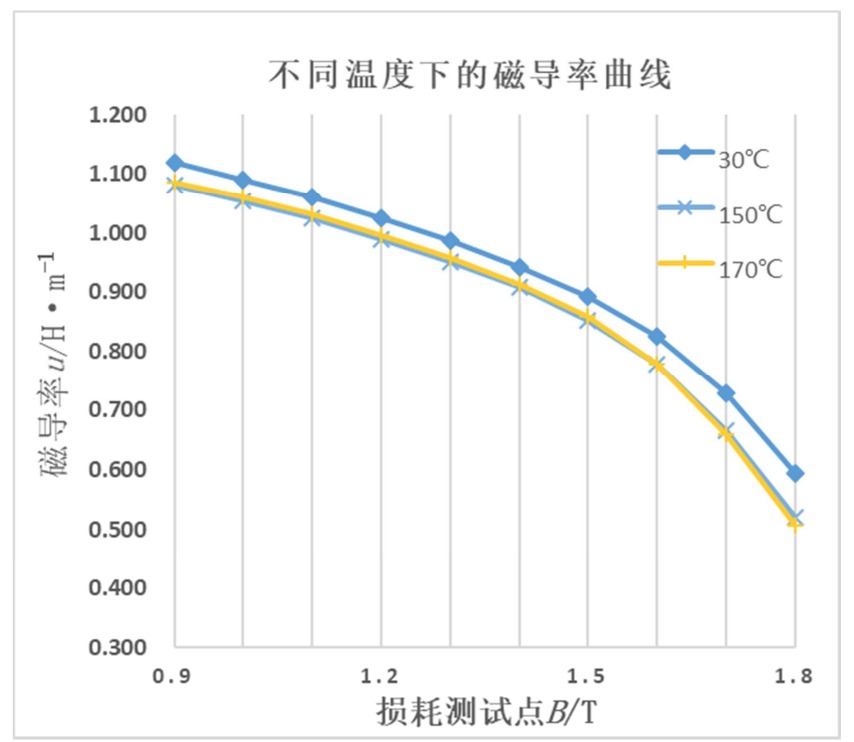

图8 $30^{\circ} \mathrm{C} 、 150^{\circ} \mathrm{C}$ 和 $170^{\circ} \mathrm{C}$ 的磁导率变化曲线。

由图中可以看出, 当温度从 $30^{\circ} \mathrm{C}$ 升高到 $150^{\circ} \mathrm{C}$ 时, 磁 导率具有下降的趋势, 在升高温度到 $170^{\circ} \mathrm{C}$ 时, 发现磁导 率开始平稳。

根据多项式拟合方法可以求出上图中 $30^{\circ} \mathrm{C}$ 损耗曲线 的拟合多项式如式 (16) 所示:

$$
\begin{gathered}
y=0.903 x^{4}+4.0359 x^{3}-6.8252 x^{2}+4.833 x-0.0493 \\
R^{2}=0.9999
\end{gathered}
$$

同理，可以通过同样的拟合方法求得 $170^{\circ} \mathrm{C}$ 时损耗曲 线对应的拟合多项式, 根据拟合多项式可以得到相应的拟 合曲线，对应的四次拟合多项式如式 (17) 所示:

$$
\begin{gathered}
y=-0.8965 x^{4}+3.8577 x^{3}-6.3356 x^{2}+4.3918 x+0.0425 \\
R^{2}=0.9999
\end{gathered}
$$

同理, 可以求出 $30^{\circ} \mathrm{C}^{\sim} 170^{\circ} \mathrm{C}$ 内其他温度下磁导率的拟 合多项式从而画出拟合曲线, 与比总损耗曲线相似，根据 实际需求, 可以根据拟合的多项式和曲线进行磁导率的估 读。

\subsection{3. 剩磁 $\mathrm{Br}$}

软磁材料的剩磁很小, 选取牌号为 $23 \mathrm{JGH}$ 的硅钢片数 据, 得到不同测试点的剩磁曲线如图9所示:

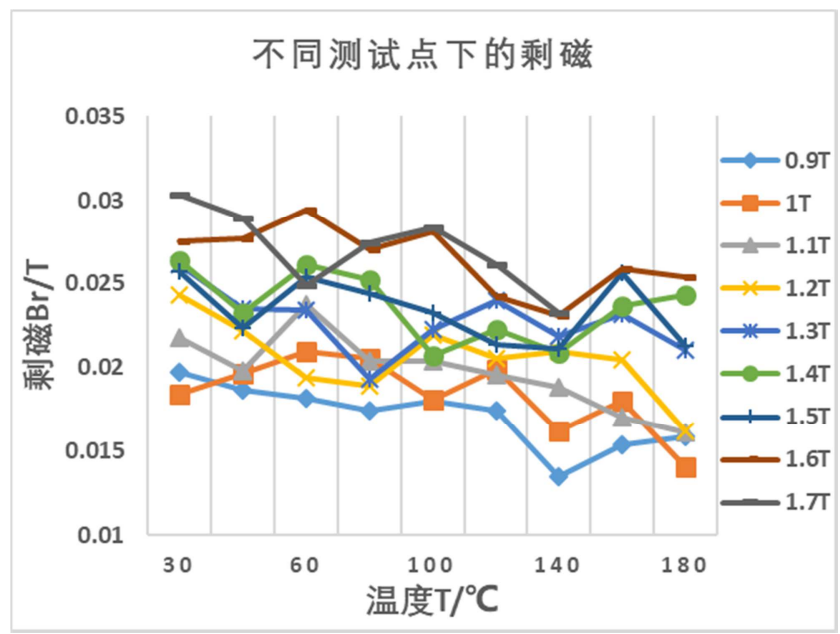

图9 不同测试点下剩磁曲线。

从图可以看出, 剩磁与温度的曲线, 很难发现直观的 规律, 但是从测试点 $\mathrm{B}$ 值来看, 测试点由 $0.9 \mathrm{~T}$ 增大到 $1.7 \mathrm{~T}$ 时, B值增大, 剩磁也跟着变大。因此, 根据这个规律, 本文通过测试数据可得到不同温度下的剩磁曲线如图 10 所示:

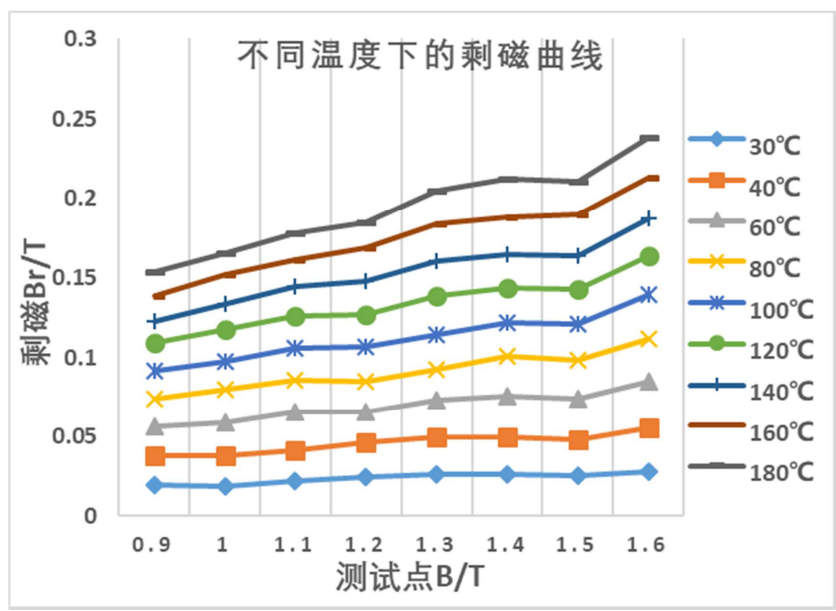

图10 剩磁随温度变化关系曲线。

从上图10可以看出, 随着温度的升高, 测试样品的剩 磁具有明显增加的趋势。相对低温度下, 测试样品随着测 试点 $\mathrm{B}$ 值的增加, 剩磁变化不明显, 随着温度的升高, 剩 
磁随着测试点 $\mathrm{B}$ 值的增加也存在增加的趋势, 且温度越高, 这种趋势越明显。

\section{4. 结论}

通过以上分析可见，本文提出了利用一维磁特性方法 环样测试法来分析磁性材料的磁特性参数, 基于环样测试 法介绍了磁特性参数的数据分析方法, 根据实验系统 TD8100实验方案, 设计制作了不同的测试样品, 对多种牌 号的硅钢片样品进行了不同温度下的磁特性参数测试实 验, 分别记录了 10 组不同测试点的比总损耗、磁导率和剩 磁参数随着温度的变化情况, 对实验数据进行了处理, 得 到与数据性质符合的拟合多项式, 从而获得了相关的磁特 性参数的变化规律, 并对实验数据出现的变化规律进行了 分析, 最终得到在温度影响下的电工硅钢磁特性参数的研 究结论。

\section{参考文献}

[1] 谢德馨, 白保东. 计算电磁学中电工钢片磁特性模型研究 的新进展 [J]. 沈阳工业大学学报, 2007, 29 (3) : 289-294.

[2] 孙纪来. 电工钢片二维磁特性测量研究[D]. 沈阳: 沈阳工 业大学, 2011。

[3] 高洁,魏剑啸,卢卫疆,阮夫明,陈新. 电工硅钢材料二维磁特 性研究及测量技术相关进展 [J]. 智能电 网,2016,4(11):1067-1072。

[4] 任志强. 电工钢片二、三维磁特性测量中的若干问题研究 [D].沈阳工业大学, 2010。

[5] Y. Li, L. Cao, C. Zhang, Q. Yang and E. Li, "Rotational Core Loss of Silicon Steel Laminations Based on Three-Dimensional Magnetic Properties Measurement," in IEEE Transactions on Applied Superconductivity, vol. 26, no. 4, pp. 1-5, June 2016.

[6] Chiampi M, Negro A,Tartaglia M. A finite element method to compute three- dimensional magnetic field distribution in transformer cores.IEEE Trans. On Magn., 1980, 16: 1413-1419.
[7] 程文锋,史正军.基于爱泼斯坦方圈测试的硅钢片磁化曲线 特性分析 [J].变压器,2017,54(12):25-28。

[8] A. M. Mohammed, T. Cox, M. Galea and C. Gerada, "A New Method for Determining the Magnetic Properties of Solid Materials Employed in Unconventional Magnetic Circuits," in IEEE Transactions on Industrial Electronics, vol. 64, no. 3, pp. 2468-2475, March 2017.

[9] Y. Zhang, Q. Li, D. Zhang, B. Bai, D. Xie and C. S. Koh, "Magnetostriction of Silicon Steel Sheets Under Different Magnetization Conditions," in IEEE Transactions on Magnetics, vol. 52, no. 3, pp. 1-4, March 2016.

[10] M. Bali and A. Muetze, "Modeling the Effect of Cutting on the Magnetic Properties of Electrical Steel Sheets," in IEEE Transactions on Industrial Electronics, vol. 64, no. 3, pp. 2547-2556, March 2017.

[11] Norio Takahashi. Notes of Short Course on Material Modeling and Measurement. September, 1999.

[12] 刘奇林. 电工钢片各向异性磁场的有限元分析 [D]. 沈阳: 沈阳工业大学, 2010。

[13] Yamamoto T, Ohya Y. Single sheet tester for measuring core losses and permeability in a silieon steel sheet.IEEE Trans.On Magn, 1974, 10(2): 157-159.

[14] Silvester P,Chari MVK.Finite element solution of saturable magnetic field problems.IEEE Trans.On Power Apparatus and Systems, 1970, PAS-89:1642-1651.

[15] Nakata T.Analysis of flux distribution of three phase three-limbed transformer core.Electrical Engineering in Japan, 1975, 95(3): 43-50.

[16] Nakata T, Ishihara Y, Yamada K, et al. Nonlinear analysis of rotating flux in the T-joint of a three phase transformer core. EPS Soft Magnetic Materials Conf., Bratislava, 1977: 57-62.

[17] Napoli A Di,Vraneo S. Anisotropic effect on magnetic field distribution in ferromagnetic region of transformer core. IEEE Conf., Boston, 1980, 4.

[18] Napoli A Di, Paggi R. A model of anisotropic grain-oriented steel. IEEE Trans on Magn., 1983, 19(4): 1557-1561.

[19] 殷春浩, 崔亦飞. 电磁测量原理及应用. 徐州: 中国矿业 大学出版社. 2008 (第二版)。 